\title{
User-centered design of a collaborative robotic system for an industrial recycling operation
}

\author{
Mouad Bounouar \\ Arts et Metiers Institute of Technology \\ LCFC, HESAM Université \\ Metz, France \\ mouad.bounouar@ensam.eu \\ Nathalie Klement \\ Arts et Metiers Institute of Technology \\ LISPEN, HESAM Université \\ Lille, France \\ nathalie.klement@ensam.eu
}

\author{
Richard Béarée \\ Arts et Metiers Institute of Technology \\ LISPEN, HESAM Université \\ Lille, France \\ richard.bearee@ensam.eu
}

\author{
Ali Siadat \\ Arts et Metiers Institute of Technology \\ LCFC, HESAM Université \\ Metz, France \\ ali.siadat@ensam.eu \\ Tahar-Hakim BENCHEKROUN \\ Conservatoire National des Arts et \\ Métiers \\ CRTD-ergonomie \\ Paris, France
}

Tahar-hakim.benchekroun@lecnam.net

\begin{abstract}
Collaborative robots (cobots) are presented as a way of business competitiveness by combining human skills with robotic advantages. The place of human operators in this context is the subject of much discussion. Including his role, his safety and his security. This study presents a methodological contribution for the management of cobotic projects. It aims to develop profitable, safe and acceptable solutions for the final users. This article presents the different steps of the design process of a collaborative robotic cell to improve a recycling laundry pods workstation. Initially, the current activity was analyzed through observations and interviews with users. Next, improvement scenarios were proposed, discussed and prioritized. After that, a feasibility study was carried out and led to technical prototyping. This was evaluated by end users to improve the solution before the investment.
\end{abstract}

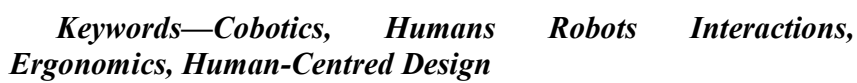

\section{INTRODUCTION}

Industrial robotics has recently undergone a new revolution thanks to the development of security technologies and the evolution of security standards governing the field. Today, robots are no longer necessarily isolated by physical barriers, but they can share the same workspace with humans! It's a new generation of robots; they are called collaborative robots. Several companies produce these new collaborative support systems. Each company usually offers several models, with different technical characteristics (repeatability, accuracy, range, maximum load, etc.) (Fig. 1).

The use of these assistance devices can be categorized into two parts in terms of interaction scenarios [1]: On the one hand, independent collaborative robots, once programmed, perform their tasks in autonomous way. Thanks to their force and presence sensors or estimators, they slow down their speed or stop to guarantee the safety of "human collaborators". Or even move dynamically to avoid collisions with surrondry obstacles [2].

On the other hand, cobots are user-guided and require a permanent human presence to be able to perform their tasks. These technological devices are presented as an innovative solution that would allow companies to be more competitive. This is achieved by benefiting from the human skills and robotic capabilities to robotize operations that have been considered recently impossible to robotize. In addition, due to their small size and ease of reprogramming, these collaborative robots/cobots are more flexible and better adapted to the fluctuating economic context.

In most of discussions about collaborative robotics, the improvement of the working conditions of future users is presented as obvious and automatic. However, some studies highlight the disadvantages generated by the introduction of new technologies and new means of production on the work conditions and therefore on the performance of the workstations concerned [3].

Interesting fields and approaches focus on end-user's needs during design projects. Non-exhaustive examples include ergonomics [4], design thinking [5], interaction design [6] and user-centered design [7]. These approaches involve end-users since the first steps of design projects, they are driven by real needs and they evaluate the progress of technical solutions with their potential users.

The challenges of this kind of innovative projects are many. Firstly, their technical complexity is often high. The safety of users is paramount, which explains why safety standards provide such an important and strong framework $[8,9,10]$. The integration of these technologies should make it possible to improve working conditions and help operators to face the difficulties encountered during their daily activities. Finally, like any industrial investment, the integration of a collaborative robotic cell must be profitable.

This study presents a methodological contribution for the management of cobotic projects. The objective is to develop profitable, safe and acceptable solutions. This case study is inspired by an industrial workstation for recycling laundry pods, by opening boxes manually after packaging or quantity non-conformities. 


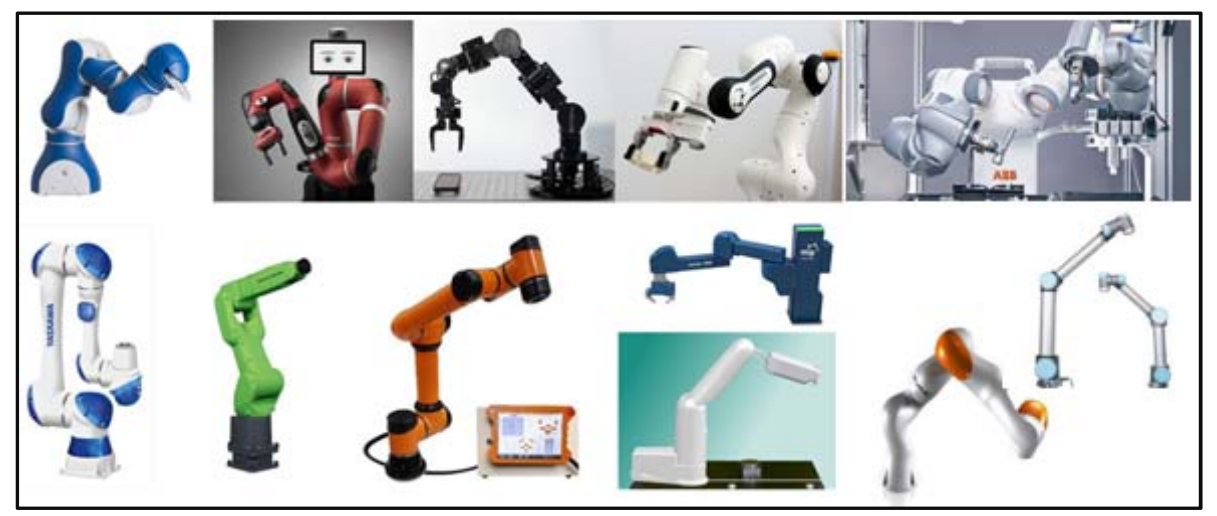

Fig. 1. Pictures of several collaborative robots.

First, we will present the context and the research methodology. Then, we will present the different steps of the methodological implementation. Then, we will discuss the results and present the perspectives of this research study.

\section{CONTEXT AND METHODOLOGY}

The industrial workstation that inspired this case study is a recycling workstation of boxes for the reprocessing of laundry pods. A job that required repetitive manual work by operators, in a chemical environment that requires the use of safety equipment such as masks and gloves. Following the appearance of a new safety standard [11] making this type of box difficult for children to open, the shapes of laundry boxes have changed by adding two push-buttons (Fig. 2), and the tasks of the operators who were in charge of recycling them have therefore become more difficult.

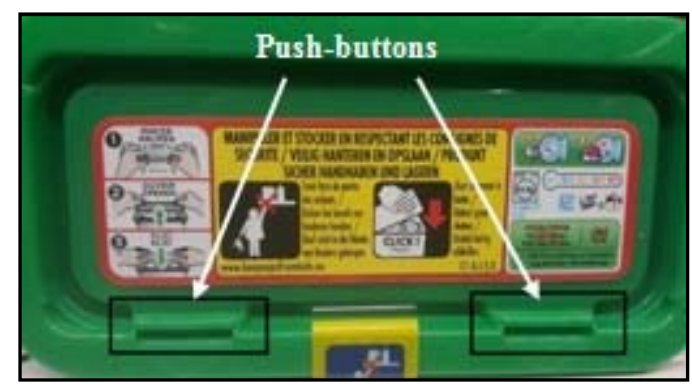

Fig. 2. Shape of the new laundry boxes.

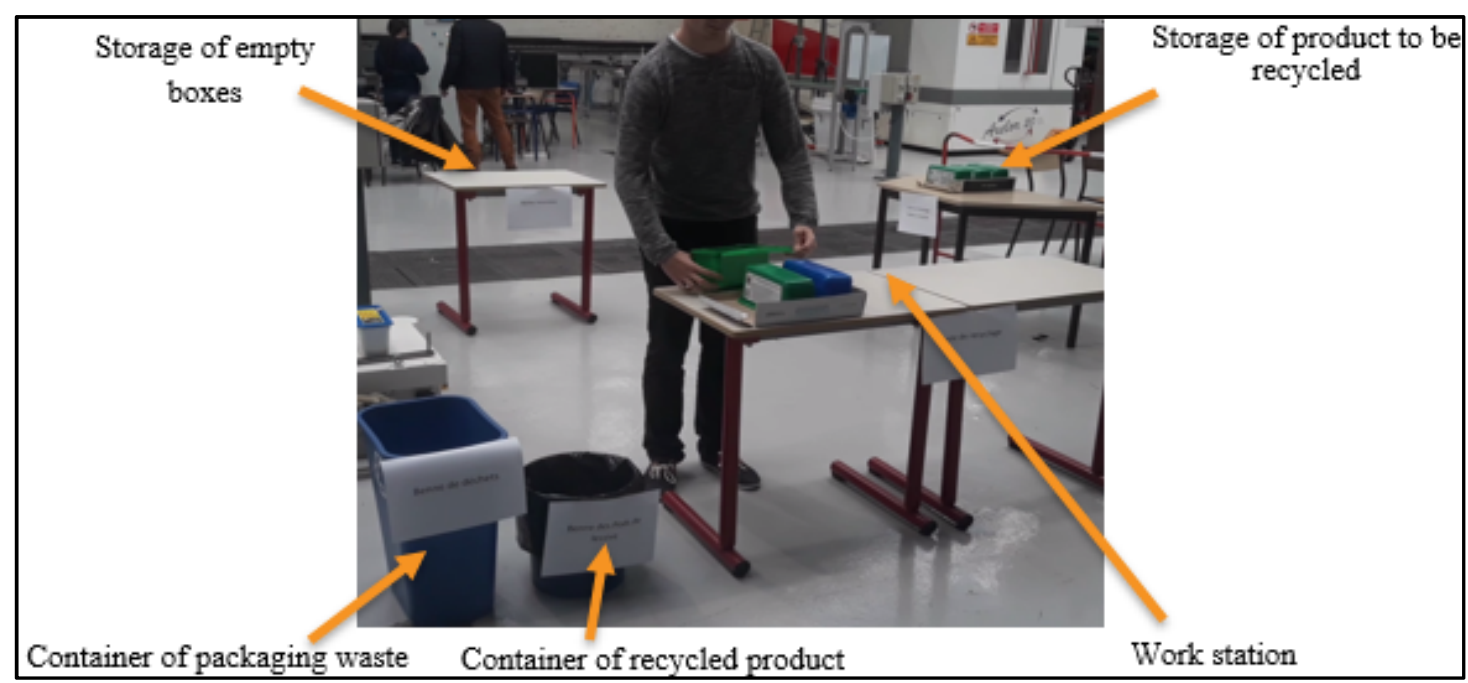

Fig. 3. Laboratory conditions of the current tasks.

\section{A. Presentation and analysis of the current activity}

The rejects from the production line are collected in cardboard boxes and sent to a recycling area. First, the operator has to pick up a batch of boxes (a cardboard of three boxes). Then, he must bring it to the workstation, open the boxes and empty the pods into a dedicated container. Once the boxes have been emptied, the operator must stack them and

place them to the storage of empty boxes (Fig. 3). cost-effective and acceptable technical support solutions this workstation, we have reproduced its tasks under laboratory conditions. This made it possible to carry out thes, evaluations and several iterations. The current with users (laboratory volunteers). Then, improvement scenarios were proposed, discussed and prioritized through collective organizational simulations. After that, a feasibility study was carried out and functional simulations led to prototyping. This was evaluated by end users to been carried out by a work team with multidisciplinary skills (robotics, ergonomics, industrial engineering).

\section{IMPLEMENTATION OF THE METHODOLOGY}


To study the problem and propose appropriate solutions for the real work environment. We started the project with a step of analysis of the current activity. This was done through observations and post-observation interviews.

After reproducing the dimensions of the real situation in the laboratory, we prepared the job description, which was inspired by the work in industrial conditions. These operations (O1 to $\mathrm{O} 8)$ consist to:

- O1: Move the cardboard boxes from the storage area to the workstation supply area;

- O2: Move a cardboard box near the containers;

- O3: Open the cardboard box;

- O4: Pick up and Open the boxes by pressing the two push buttons;

- O5: Place the laundry pods in the appropriate container;

- O6: Stack the empty boxes on the table;

- O7: Dispose of the cardboard in the waste container;

- O8: Move the stacked empty boxes to the storge of empty boxes area; Fig. 4.

The spatial organization of these operations is shown in

We asked voluntary operators to carry out the recycling operations for 15 minutes. These trials were observed and followed by systematic interviews with all volunteers. These interviews were aimed at finding out, among other things: Whether there are any non-intuitive operations or painful operations, time pressures, work environment-related problems to be identified or improvements to be proposed.

Based on this activity analysis step, the "O4" operation involving the opening of boxes has proved to be the most constraining operation. Operators must work both quickly (productivity target) and precisely (press both buttons to open the box). This is particularly visible through the variety of techniques applied to perform this operation. Sometimes the operator places the box on his workstation and presses both hands on the two buttons to open it, other times he carries the box and presses with both hands on the two push buttons, or he carries it with one hand and presses on the two buttons with the other hand (Fig. 5).

Post-observation interviews confirmed this observation. In fact, most operators noted that this operation is not fluent unlike moving operations of boxes to be recycled, empty boxes and waste.

During this step, the productivity of each volunteer operator was also measured. The average productivity is about 6 recycled boxes per minute.

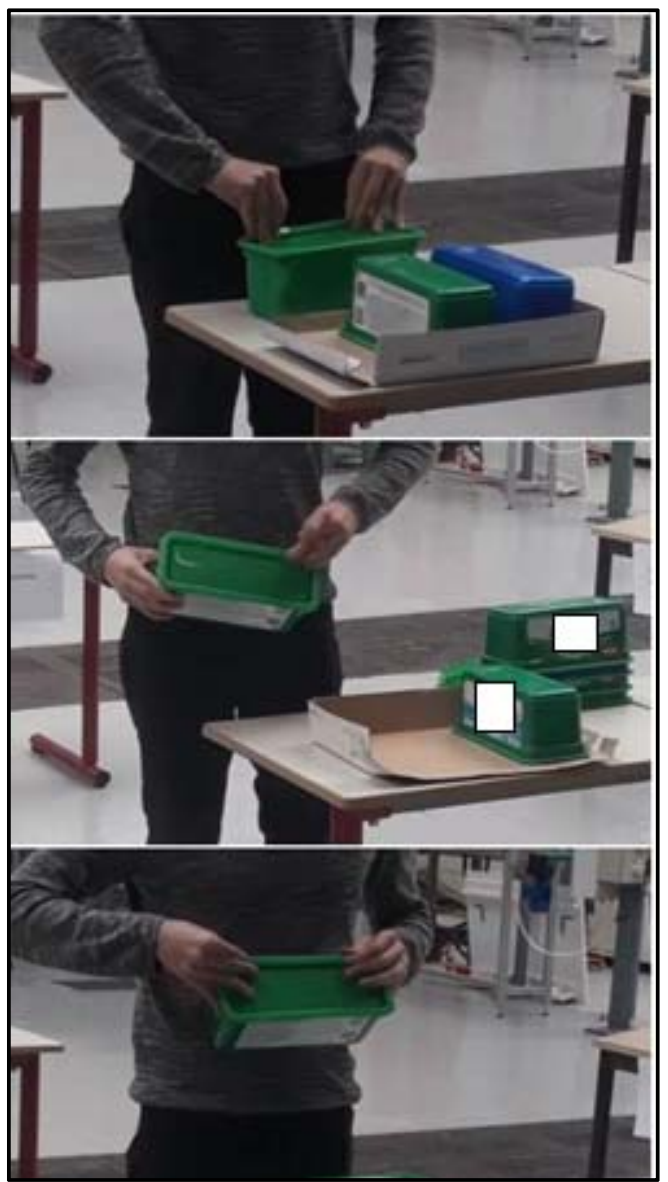

Fig. 5. The variety of the observed techniques of the box opening operation.

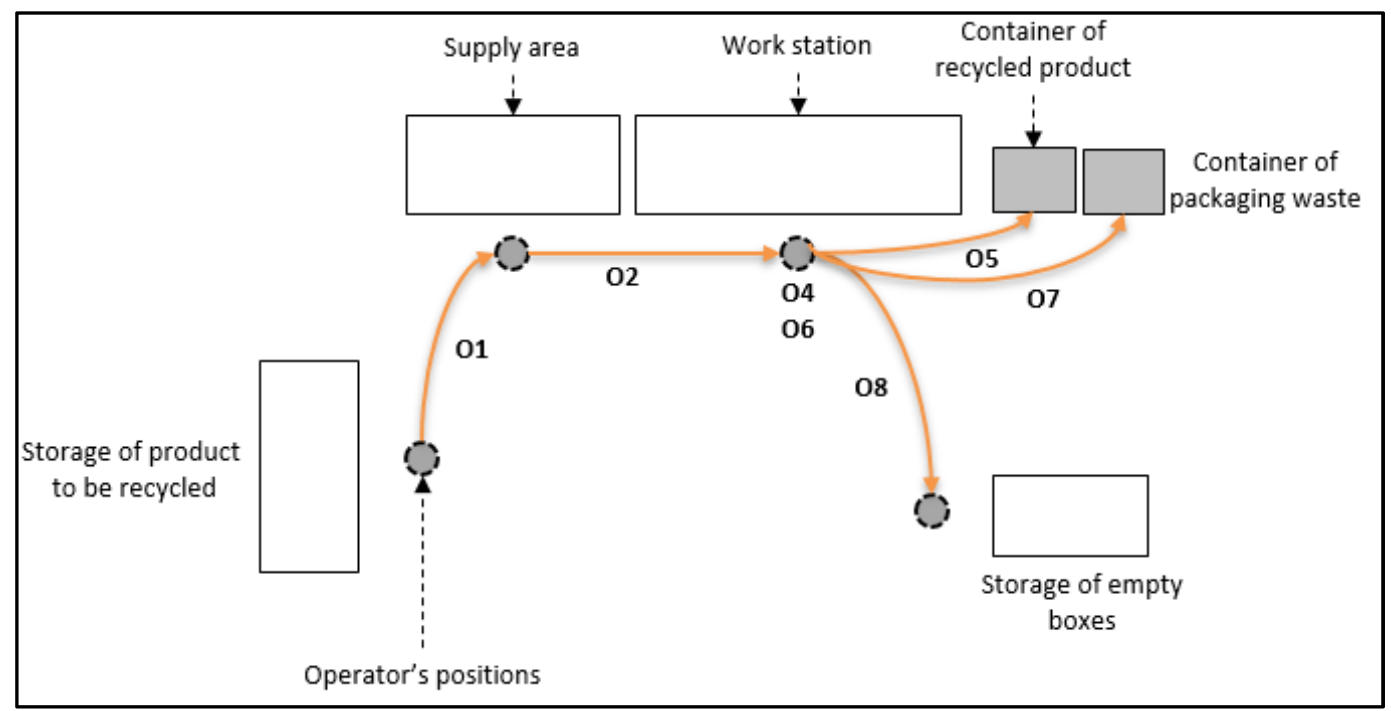

Fig. 4. The spatial organization of operations. 


\section{B. Ideation and collective evaluation of the proposed solutions}

Based on the results of the activity analysis, an ideation phase was conducted to propose solutions in order to assist operators during the recycling of laundry boxes and to remedy the difficulties encountered precisely when opening these boxes. In this regard, two ideas have been proposed:

- The design and manufacture of a manual tool to help opening the boxes (considering their new shape);

- The development of a collaborative robotic cell, where the robot will handle the most constraining operations.

Discussions made it possible to compare the two tracks and favored the second proposal. In fact, even with a manual tool to help open boxes, operators will always be required to carry out the same steps, and maybe the same gestures with a level of complexity not necessarily lower than before. The team therefore concentrated on finding technical solutions for opening boxes using a collaborative robot. The following section provides a brief overview of the feasibility study to robotize this operation.

\section{Technological feasibility study and functional simulations}

The challenge of this step was to design the technical solution to open the laundry boxes. The choice of the robot is not the most important issue during a robotic cell design process. However, the choice or the design of the End Effector is not always easy. In fact, no ready-to-use effector reference was found for opening this type of boxes. Several experiments have led to the following solution principle: the robot will clamp the box on the bottom side and open it through contact with a fixed support. Picture B in Fig 6 shows the chosen principle due to its efficiency.

Mechanical parts have been designed and manufactured (with a 3D printer) to facilitate the robot's operations. These include a part for cutting the adhesive tape closing the boxes (Fig7.A), a support for the stacked boxes (Fig7.B) and a guidance support to ensure the exact position of the boxes to be recycled (Fig7.C) in the robot's area of application.

To better benefit from the deployment of this robot, other tasks have been allocated to it (Table 1). This task allocation between the operator and the robot was proposed, evaluated and validated by the design team.

After these steps of organizational design (task allocation between Human and Robot) and technological feasibility study, an industrial prototype was developed to test the relevance of the solution.

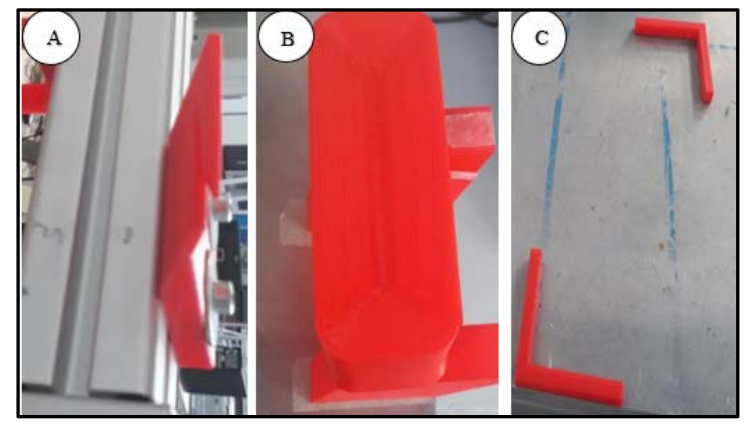

Fig. 7. Mechanical parts designed to improve the solution.

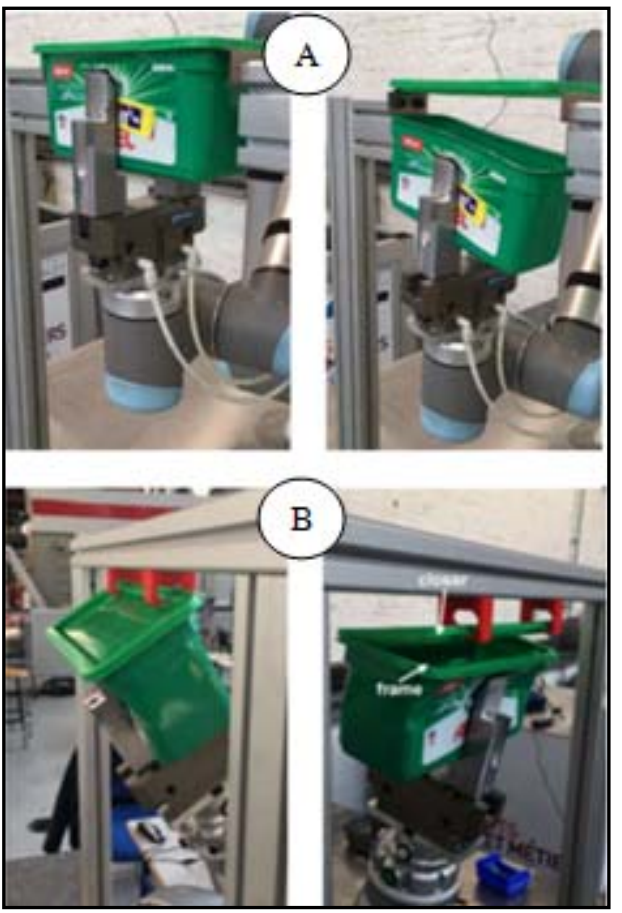

Fig. 6. Examples of the solution principles tested to open the boxes.

TABLE 1. PROPOSED TASKS ALLOCATION

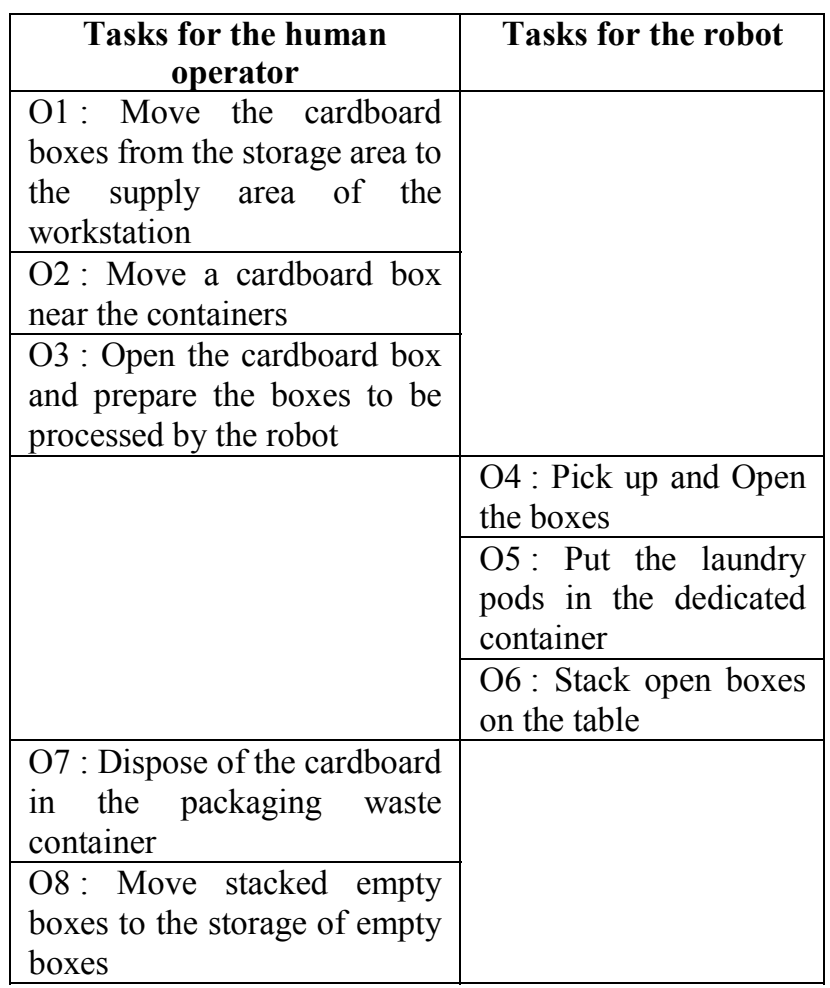

D. Industrial prototyping and assessment of the acceptability

As illustrated (Fig. 8), the industrial prototype has made it possible to simulate the expected future of the laundry box recycling workstation. First, the human operator prepares the laundry boxes in a specific location, the robot comes and picks them up (Fig. 8, part 1). Then the robot cuts the tape from the box (Fig. 8, part 2), opens the box (Fig. 8, part 3), and empties the box into a dedicated container (Fig. 8, part 4). Subsequently, the robot stacks the empty boxes (Fig. 8, 
part5) and the operator prepares other boxes at the same time so that the cycle restarts.

The characteristics of the operator's work have changed significantly. Introducing a technology is also a change on a socio-organizational system [12]. In order to avoid rejection by users, the acceptability of the new system has been discussed.

Many theoretical models have aimed to specify the determinants of technological acceptance $[13,14]$, the most widely used is the Technology Acceptance Model (TAM) [15]. This model explains the acceptability process by two main subjective factors: perceived utility and perceived ease of use. These two factors would influence attitudes and intentions to use a new technology or tools.

For our case, the utility is clear and recognized by all the people who tested the solution. In fact, the robot handles the boxes opening operation successfully which was the most constraining task.

Under laboratory conditions, the proposed solution was user-friendly, but basic operator training is required to learn how to use the robotic cell.

A more formal evaluation of human conditions is planned under industrial conditions as the solution developed will be installed at an industrial company within the next two months. This assessment will be carried out using a revised form of the System Usability Scale [16], one of the most widely used scales aiming to anticipate users' usability.

Regarding profitability, the simulations with the developed prototype were an opportunity to measure the productivity of the new system, it was about 3 recycled boxes per minute.

\section{DISCUSSION}

The implementation of a new technology represents an important change in the organisation and activity of operators. In this case study the role of operator has completely changed. In fact, he used to recycle laundry boxes, now he will be managing and supervising a laundry box recycling cell. This type of change, if not chosen by end users, will not be easily accepted and implemented.

In order to prevent rejection by users, the acceptability of the new system must be discussed and addressed at an early stage of the design project.

Early in the project, the team must focus on understanding the current activity by analysing the tasks of the operators through observations, interviews and analysis of documents related to the workstation. This step should allow to identify sources of variability, any encountered difficulties and the strategies to manage them in the daily work.
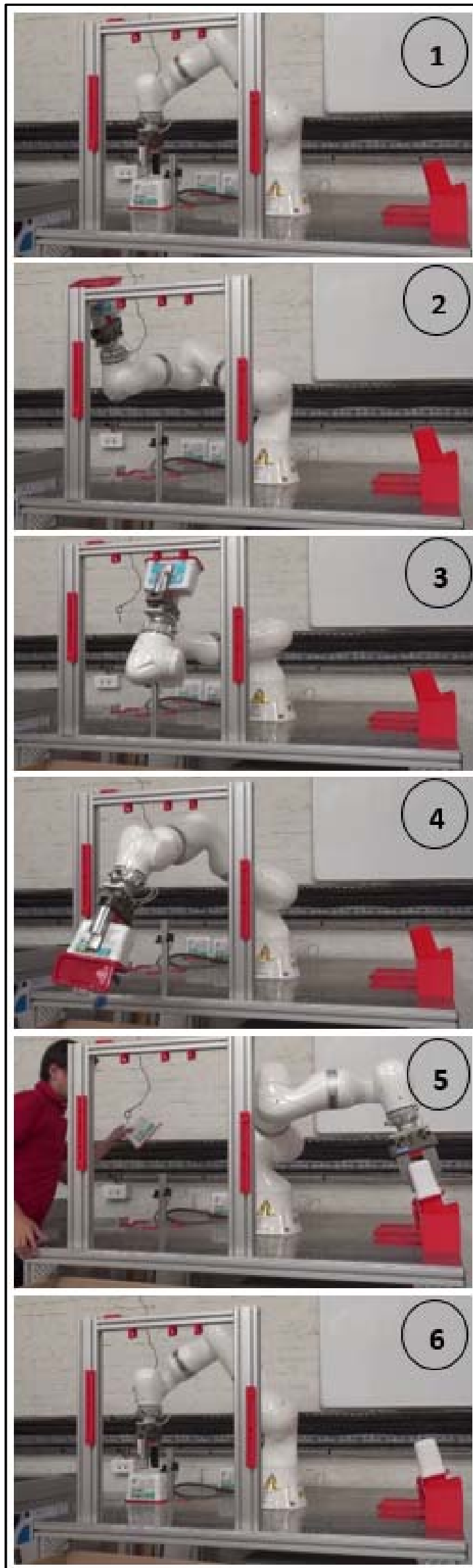

Fig. 8. Industrial prototype of the collaborative robotic cell for the laundry box recycling operations. 
In our case, the simulations of the manual activity organized in the laboratory made it possible to detect one of the most constraining operations, and to identify possible improvements for the workstation. However, observations and interviews with real operators who have experiences in the workplace could lead to better results.

The design steps must be participative and iterative. Starting with a stage of ideation of solutions and Humansrobots collaboration scenarios, which must be discussed, evaluated and prioritized. Then, the favoured scenarios must be studied in order to propose technological solutions. These solutions, once accepted by the organization (final users, mangers and direction), must be simulated and prototyped in order to evaluate their potential impacts on performance and on human conditions. In our case, improvements of the collaborative robotic cell on human conditions have been satisfactory and appreciated by all volunteer operators.

Regarding productivity, the simulations estimated the new workstation at around 3 recycled boxes per minute. This represents a degradation of $50 \%$ compared to the initial phase ( $100 \%$ manual, 6 boxes per minute). However, this result is not very representative because in the industrial conditions, a buffer stock area with a conveyor to the recycling station is expected, and will free up time for the operator so that in addition to monitoring and managing the recycling workstation, he would be able to handle other tasks.

The collaboration scenario does not involve any physical contact between the human and the robot during the production time. The safety of users will be ensured by a safety-rated monitored stop [10]. This consist on stopping the robot if a human enters its collaborative workspace. It is made using sensors to detect human presence.

The evaluation phase on the industrial site should provide the feedback from the real operators about this solution. Then the design team will iterate until reaching a more appropriate version of the solution.

\section{CONCLUSION}

Collaborative robotics presents several perspectives for the future. It would allow companies to be more competitive by monitoring fluctuations in customer demand, by robotizing operations that have been considered recently impossible to robotize, and by reducing repetitive and constraining tasks. However, the integration of a collaborative robot is not neutral. It involves a change in the work organization, a reassignment of operators, a change in teamwork, etc.

Considering final users during the design projects could lead to combining productive performance, health, safety and development of skills. In this article, the current activity was analysed through observations and interviews with users (laboratory volunteers). Next, improvement scenarios were proposed, discussed and prioritized. After that, a feasibility study was carried out and led to technical prototyping. This was evaluated by end users to improve the solution before the investment.

The multidisciplinary aspect is one of the main keys for success of cobotization projects, the design team must include at least final users, managers (production, quality, etc.), direction representative, an ergonomist and a robot integrator.

The iterative evaluations would allow to anticipate the different potential impacts of the cobotic cell on performance, on human conditions and on the collective work.

Future work will focus on the development of a multidisciplinary methodological framework for the management of cobotic system design process. This methodological framework would assist designers to consider the various aspects related to such a new project (technical, economic, human).

\section{REFERENCES}

[1] M. Bounouar, R. Bearee, T-H. Benchekroun and A. Siadat, "Etat des lieux de la cobotique industrielle et de la conduite de projet associée," presented at the 16th S-mart colloque. Les Karellis, France, Apr 3-5, 2019.

[2] M. Safeea, P. Neto, and R. Bearee, "Efficient Calculation of Minimum Distance Between Capsules and Its Use in Robotics," IEEE Access, vol. 7, pp. 5368-5373, 2019.

[3] A. Garrigou, J.-F. Thibault, M. Jackson, and F. Mascia, "Contributions et démarche de l'ergonomie dans les processus de conception," Perspectives interdisciplinaires sur le travail et la santé, no. 3-2, pp. 1-18, Jan. 2001.

[4] F. Guérin, A. Laville, F. Daniellou, J. Duraffourg , and A. Kerguelen, Comprendre le travail pour le transformer: la pratique de l'ergonomie. Montrouge: ANACT, 1991.

[5] F. Mathieu and Hillen Véronique, Le design thinking par la pratique: de la rencontre avec lutilisateur à la commercialisation dun produit innovant pour les séniors. Paris: Eyrolles, 2016.

[6] P. Savioja, M. Liinasuo, and H. Koskinen, "User experience: does it matter in complex systems?," Cognition, Technology \& Work, vol. 16, no. 4, pp. 429-449, May 2013.

[7] Ergonomics of human-system interaction, ISO 9241-210:2010 (2010), Part 210: Human-centred design for interactive systems. International Organisation for Standarization.

[8] Robots and robotic devices, Safety requirements for industrial robots, ISO 10218-1:2011 (2011), Part1: Robots. International Organization for Standarization.

[9] Robots and robotic devices, Safety requirements for industrial robots, ISO 10218-2:2011 (2011), Part 2: Robot systems and integration. International Organization for Standarization.

[10] Robots and robotic devices-Collaborative robots, ISO/TS 15066:2016 (2016). International Organization for Standarization.

[11] Classification, labelling and packaging of substances and mixtures, Rules (European Union) NO 1297/2014 (2014). European commission of Dec 5, 2014

[12] M.-E. B. Chaumon, "L'acceptation située des technologies dans et par l'activité : premiers étayages pour une clinique de l'usage," Psychologie du Travail et des Organisations, vol. 22, no. 1, pp. 4-21, 2016.

[13] M. T. Dishaw and D. M. Strong, "Extending the technology acceptance model with task-technology fit constructs," Information \& Management, vol. 36, no. 1, pp. 9-21, 1999.

[14] D. L. Goodhue and R. L. Thompson, "Task-Technology Fit and Individual Performance," MIS Quarterly, vol. 19, no. 2, pp. 213236, 1995.

[15] F. D. Davis, "Perceived Usefulness, Perceived Ease of Use, and User Acceptance of Information Technology," MIS Quarterly, vol. 13 , no. 3, pp. 319-340, 1989.

[16] J. Brooke, "SUS: A retrospective," Journal of usability studies, vol. 8, no. 2, pp. 29-40, 2013. 